\title{
Effect of Nifedipine on Memory Impairment Induced by Repetitive Spreading Depression
}

\author{
Mahmoud Lotfinia ${ }^{1,2}$ ", Ahmad Ali Lotfinia ${ }^{1,3}$, Babak Khodaie ${ }^{1,3}$, Milad Ahmadi, ${ }^{1,}$, Maryam Jafarian ${ }^{1,4}$ \\ ${ }^{1}$ Shefa Neuroscience Research Center, Khatam-al-Anbia Hospital, Tehran, Iran. \\ ${ }^{2}$ Shahid Beheshti University of Medical Sciences, Tehran, Iran. \\ ${ }^{3}$ Faculty of Veterinary medicine, Karaj Branch, Islamic Azad University, Karaj, Iran. \\ ${ }^{4}$ School of Advanced Technologies in Medicine, Tehran University of Medical Sciences, Tehran, Iran.
}

\section{Received: 12 Oct, 2013

\section{A BSTRA CT}

I

ntroduction: Spreading depression (SD) is known by transient loss of spontaneous and evoked neuronal activity and changes in ionic, metabolic and hemodynamic characteristics of the brain. It has been shown that repetitive SD produced memory deficits in juvenile rats. Furthermore, the role of $\mathrm{Ca}^{2+}$ channels on induction and propagation of SD was investigated by several scientists. The aim of the present study was to study the role of a $\mathrm{Ca}^{2+}$ channel-blocker, nifedipine, on memory deficits induced by repetitive SD. Materials and Methods: Wistar rats (60-80gr) were divided into 5 groups and nifedipine (1 $\mathrm{mg} / \mathrm{kg}$ ) was administrated weekly for 4 weeks in SD group. SD was also induced weekly for four weeks by $\mathrm{KCl}(2 \mathrm{M})$. Retrieval of spatial memory was evaluated by T-maze memory test. Results: The T-maze test demonstrated that memory was impaired in SD group. The memory retrieval significantly improved by application of nifedipine. Conclusion: This study suggests the possible role of calcium channels in memory impairments following repetitive SD.

\section{Key words:}

1.Cortical Spreading Depression

2. Nifedipine

3. Memory

* Corresponding Author: Mahmoud Lotfinia

E-mail:mdla617@yahoo.com 


\section{بررسى تأثير داروى نيفدييين بر تخريب حافظه ى ناشى از القاى مكرر مههار منتشر شونده}

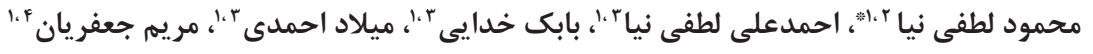

'مركز تحقيقات علوم اعصاب شفا، بيمارستان خاتم الانبياء، تهران، ايران.

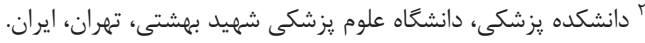

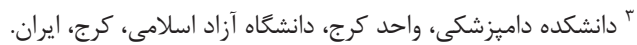

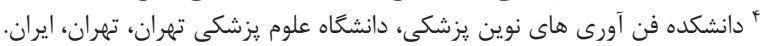

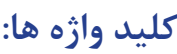

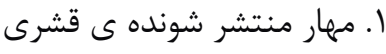

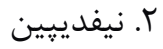

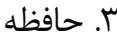

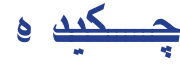

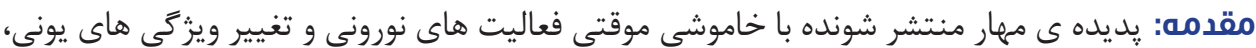

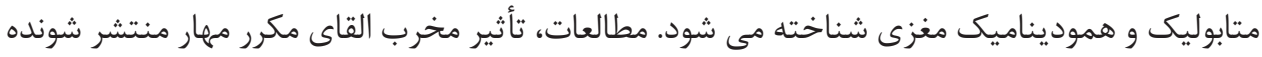

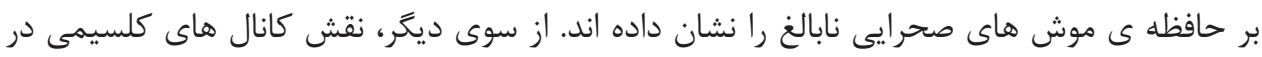

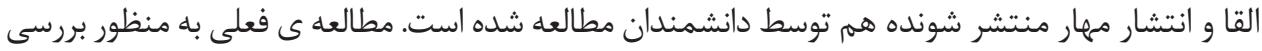

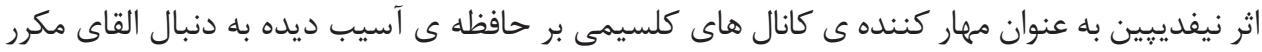

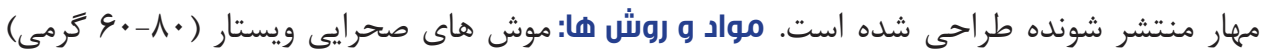

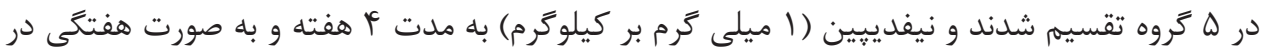

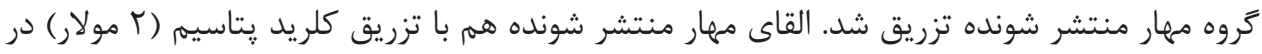

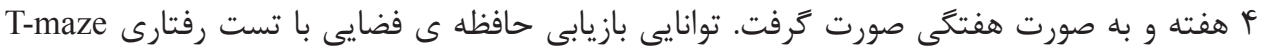

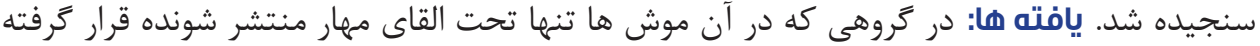

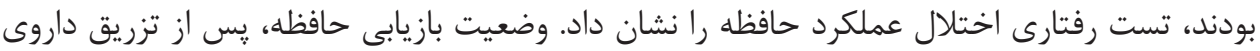

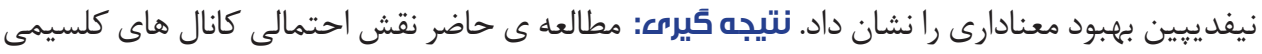
در تخريب حافظه ناشى از القاى مكرر مهار منتشر شونده رادي ران نشان مى دهر نهد.

" نويسنده مسئول: محمود لطفى نيا

آدرس الكترونيكى: mdla617@yahoo.com 


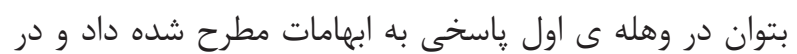
نهايت همم قدمى در مسير شناخت SD و اثرات آن بر مغز بره دات داشت

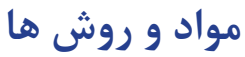
حيوانات و َروه هاى مورد آزمايش هو مايش

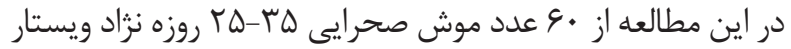

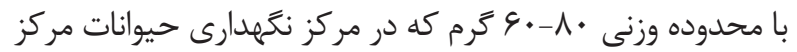

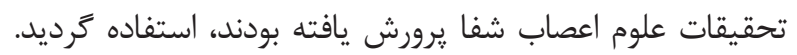

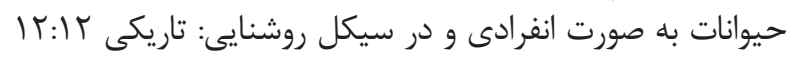

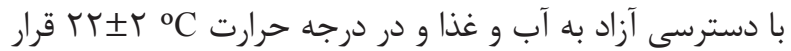

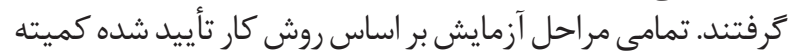

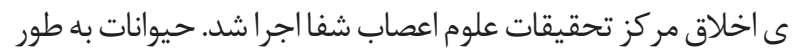

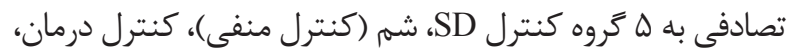
درمان و كنترل حلال دارو تقسيم شدند.

تزريق كلريد يتاسيم و داروى نيفدييين دركروه هاى مختلف به

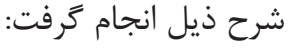

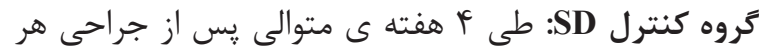

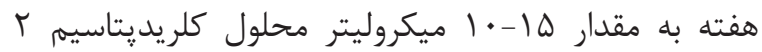

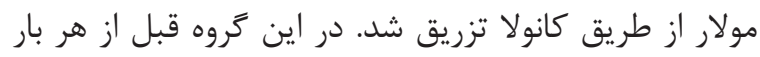

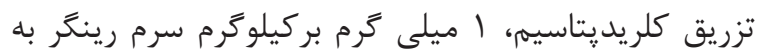
صورت داخل صفاقى تزريق كرديد.

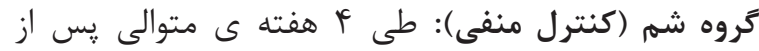

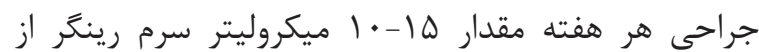

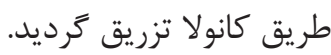

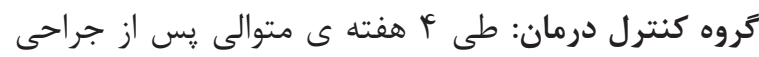

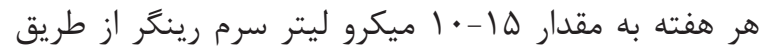

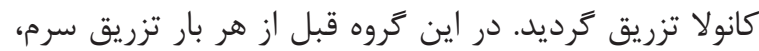

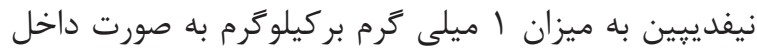

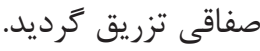

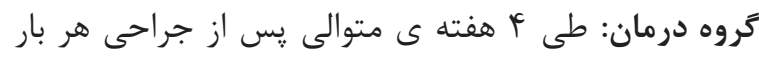

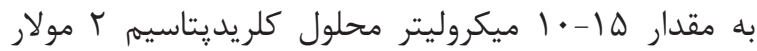

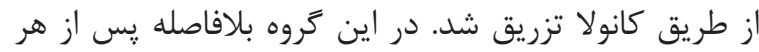

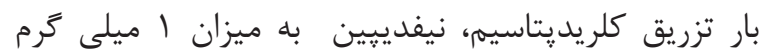
بر كيلوكرم به صورت داخل صفاقى تزريق ترئ نرديد.

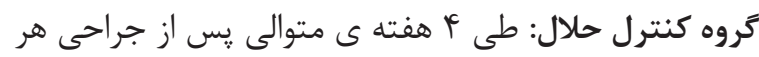

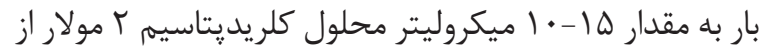

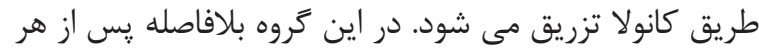

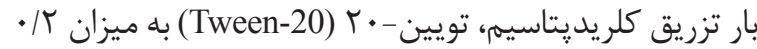

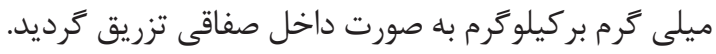

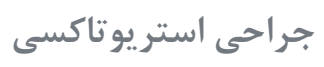

در ابتدا موش ها با استفاده از يك ترازوى حساس وزن شدئ شدئ

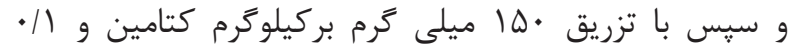

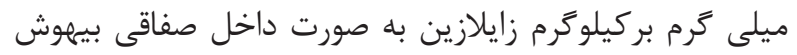

${ }^{1}$ Spreading Depression

\section{مقدمه}

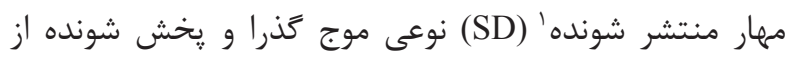

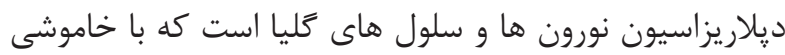

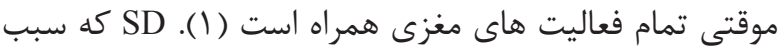

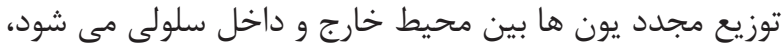

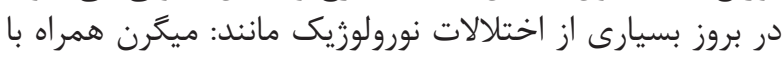

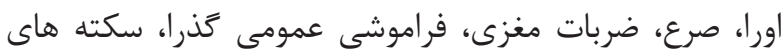

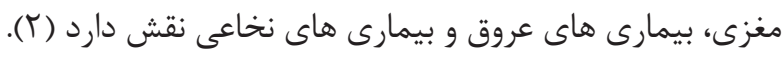
عوامل مختلفى نظير تحريكات الكتريكى، تحريكات مكانيكى،

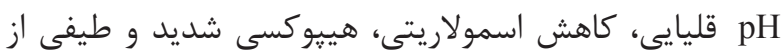

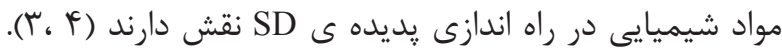

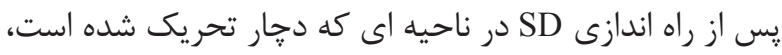

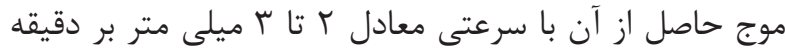

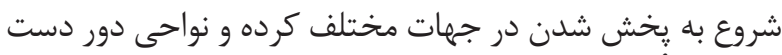
را تحت تأثير قرار مى دهن دهد (ه).

شواهد متعددى در مورد نقش يون كلسيم در روند انتشار و آسيب

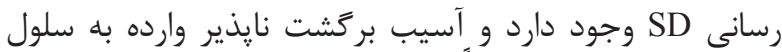

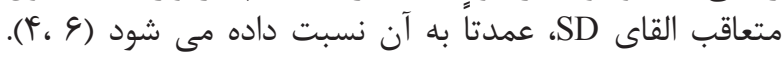

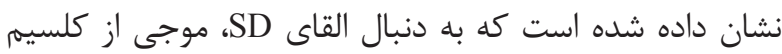

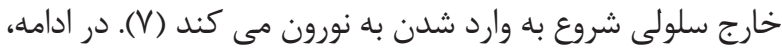

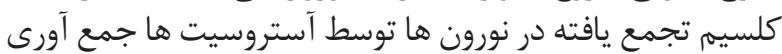

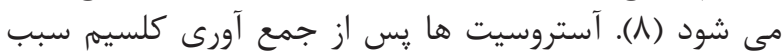

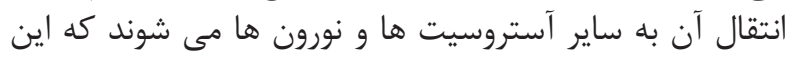

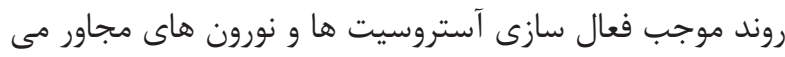

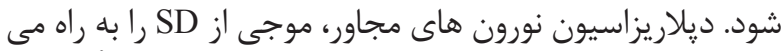

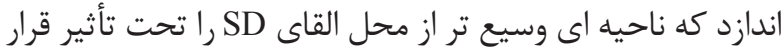

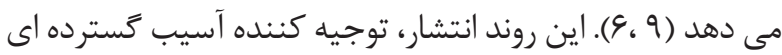

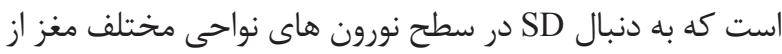

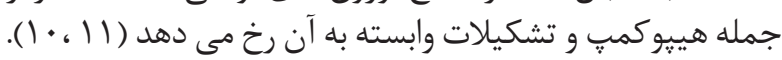

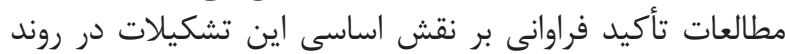

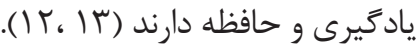

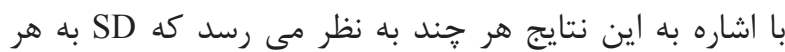

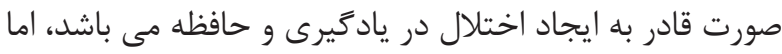

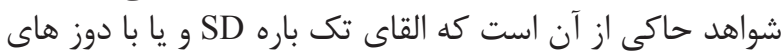

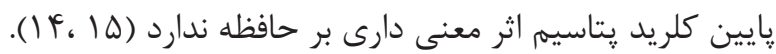

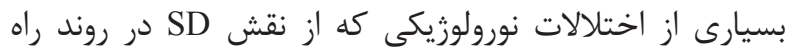

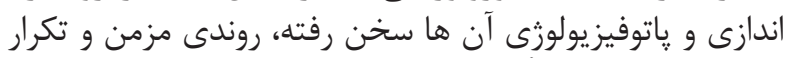

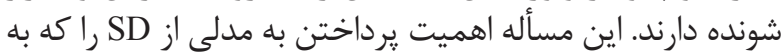

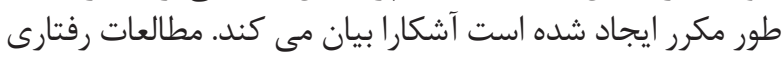

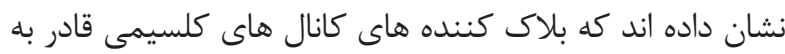

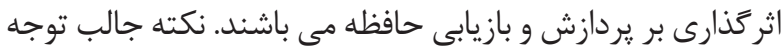

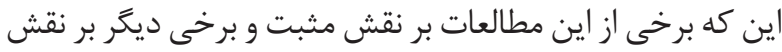

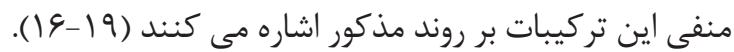

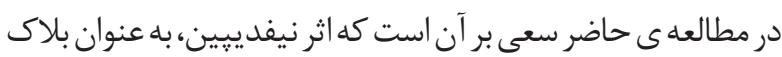

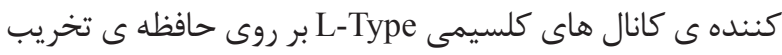
شدهى ناشى از القاى مكرر SD سنجيده شود تا از اين منظر شايد 
صورت داخل صفاقى به موش ها تزريق شد. همجنين براى كروه

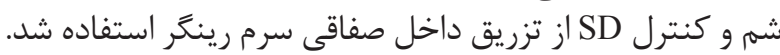
داروى مورد استفاده در روز آزمايش تهيه كردي ترديد.

$$
\text { آزمون رفتارى دورى مورد اسناهن }
$$

عملكرد حافظه ى موش هاى مورد مطالعه به وسيله ى تست

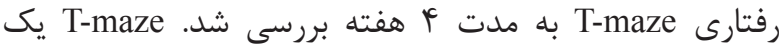

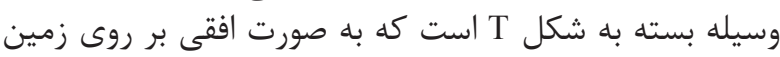

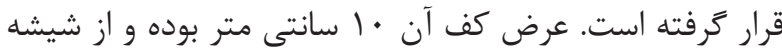

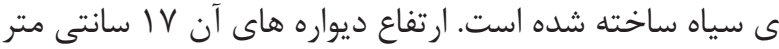

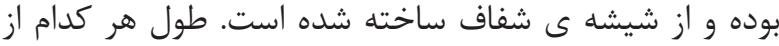

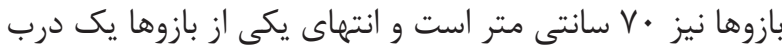

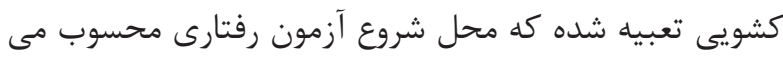

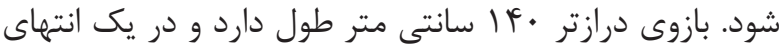

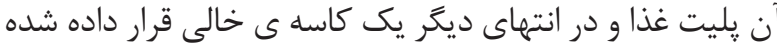

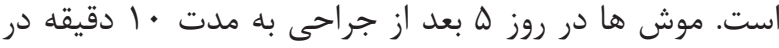

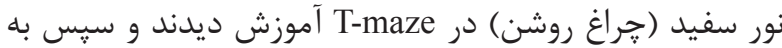

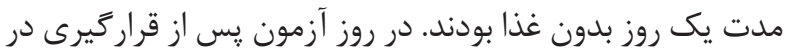

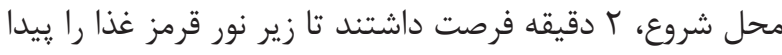

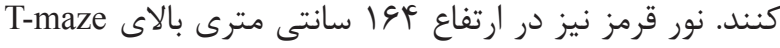

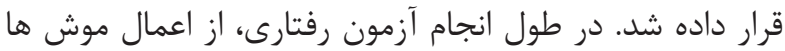

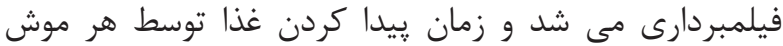

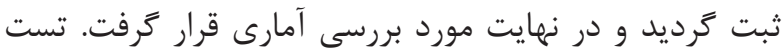

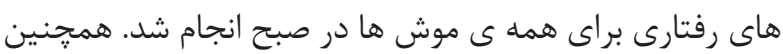

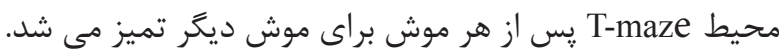

$$
\text { آناليز آمارى }
$$

نتايج به دست آمده از آزمون رفتارى توسط نرم افزار مورد

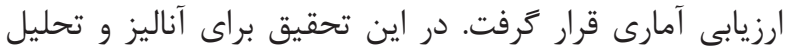

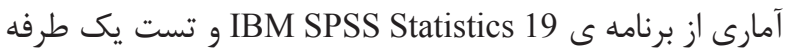
ANOVA

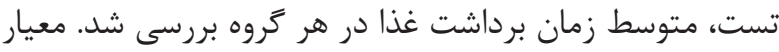

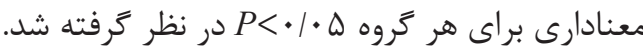

هافته ها

بررسى آمارى نتايج آزمون رفتارى نشان دهنده ى اختاليال در

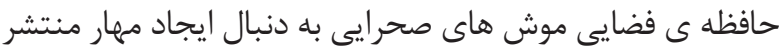

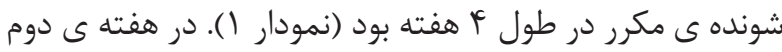

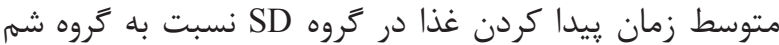

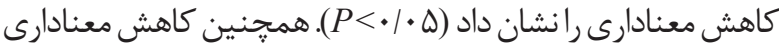

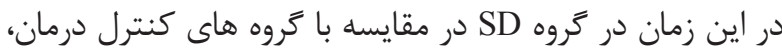

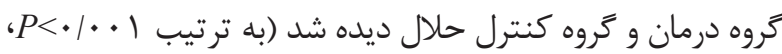

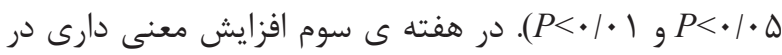

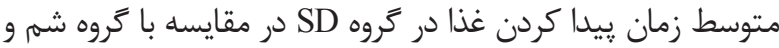

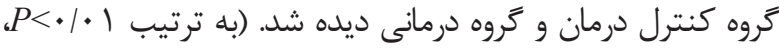
SD

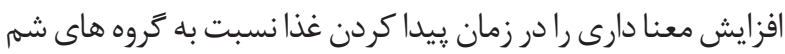

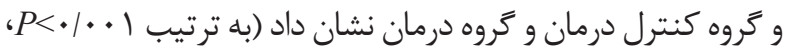

${ }^{2}$ Stereotax

${ }^{3}$ Stoelting Instruments, USA
شدند. يُ از بيهوشى سر حيوان در دستكاه استريوتاكس

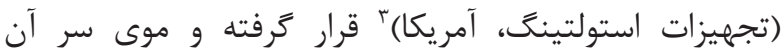

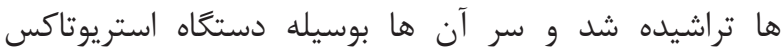

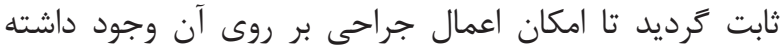

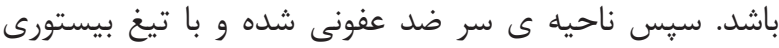

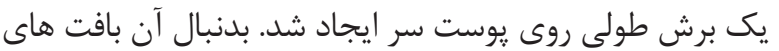

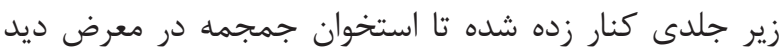

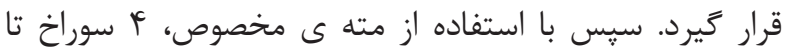

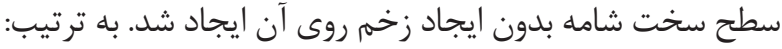

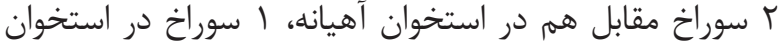

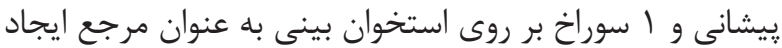

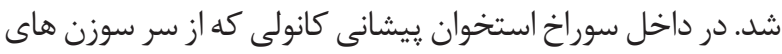

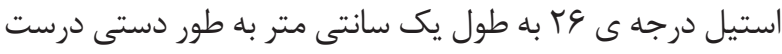

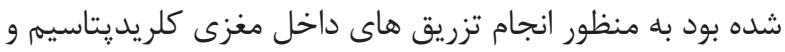

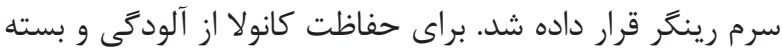

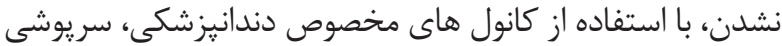

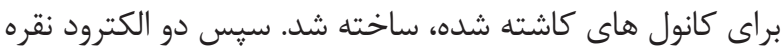

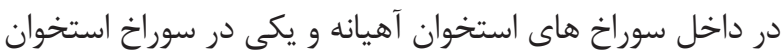

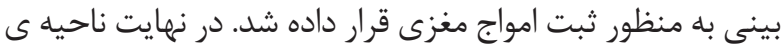

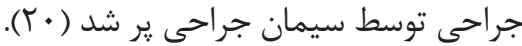

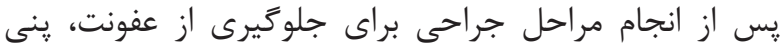

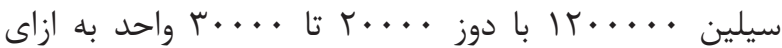

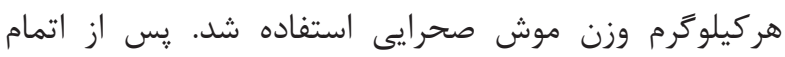

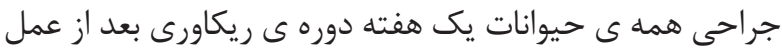

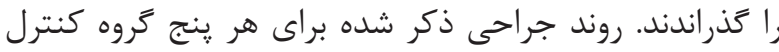

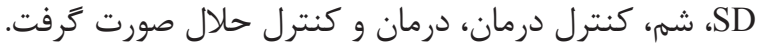

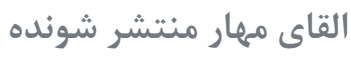

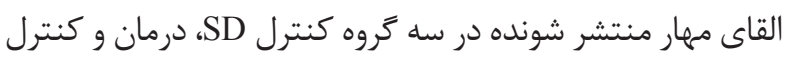

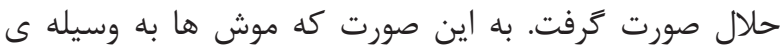

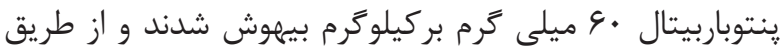

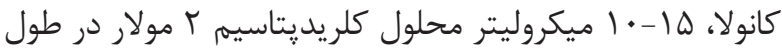

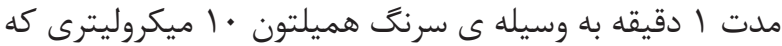

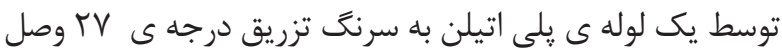

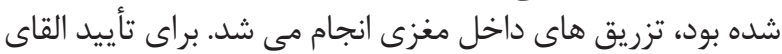

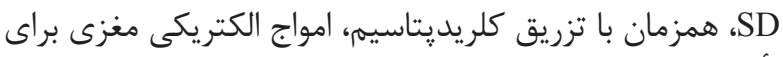

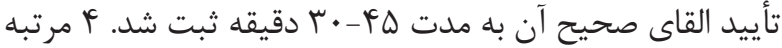

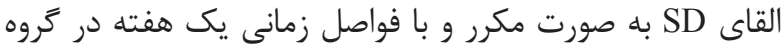

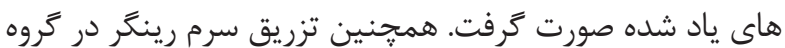

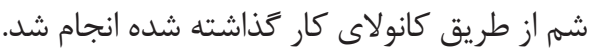

داروى نيفدييين (Sigma) كه به عنوان يك آنتاكونيست كانال هاى

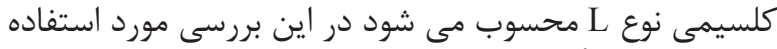

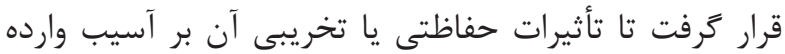

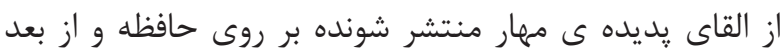

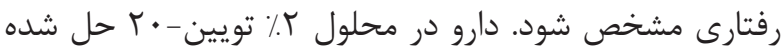

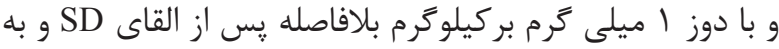




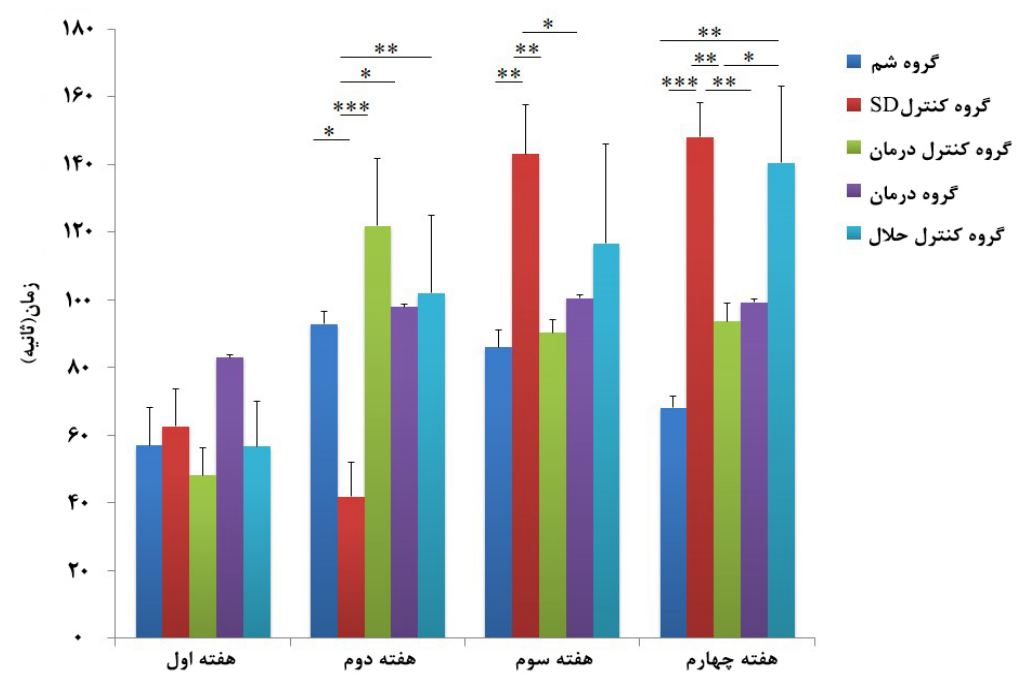

\begin{abstract}
ثم

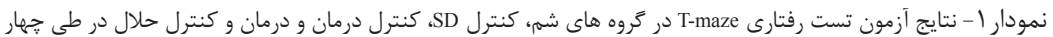

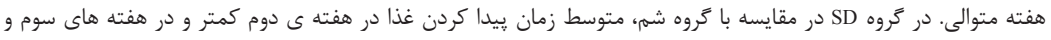

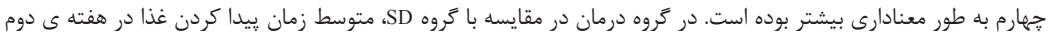

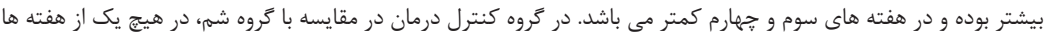

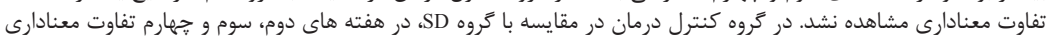

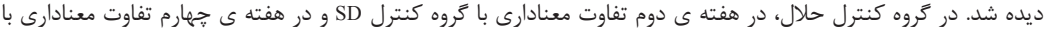

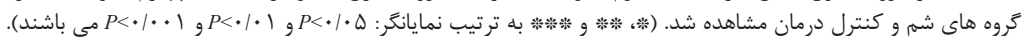

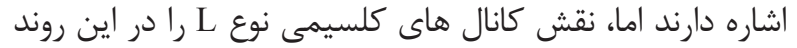

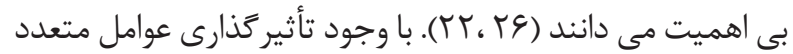

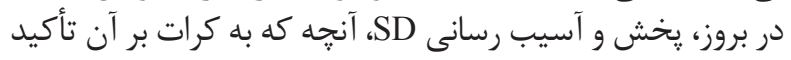
شده است، يون كلسيم و كانال هاى كلسيمى مى باشد.

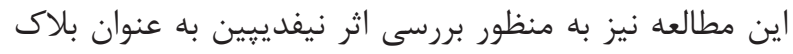

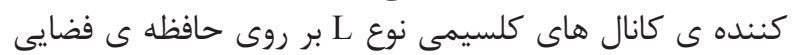

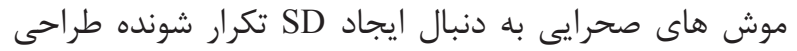

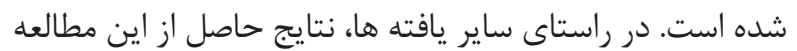

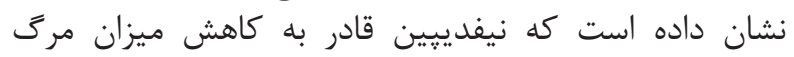

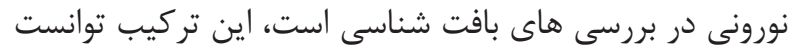

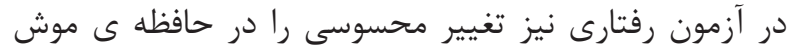

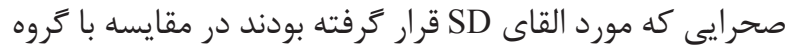

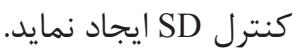

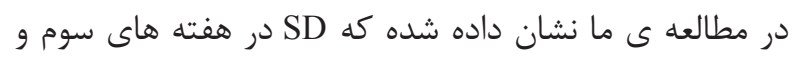

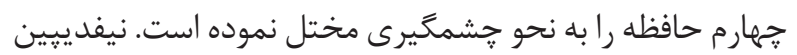

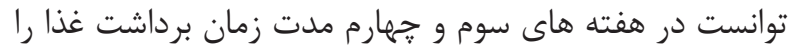

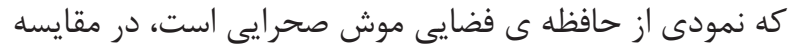

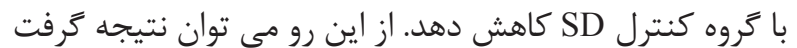

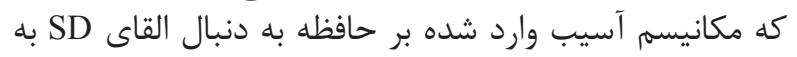

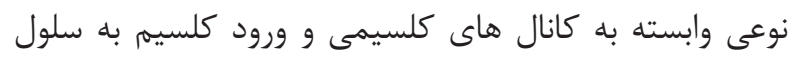
مى باشد.

نتايج اين مطالعه بر اهميت نقش كانال هاى كلسيمى به عنوان

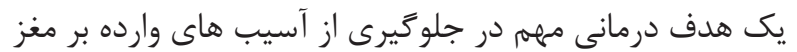
به دنبال وقوع يديده ى SD تأكيد دارد.

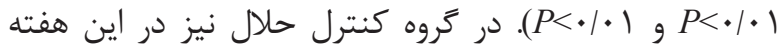

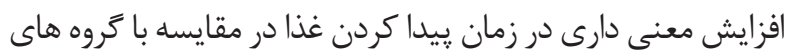

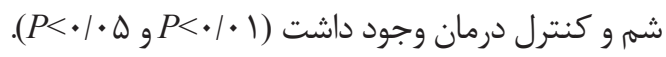

\section{بحث و نتيجه تيرى}

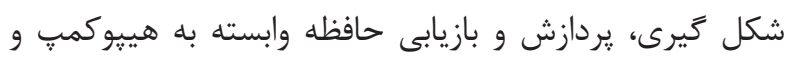

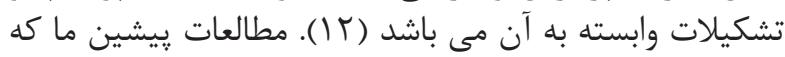

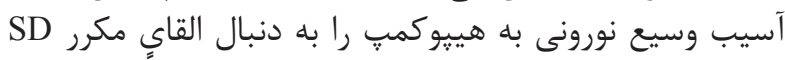

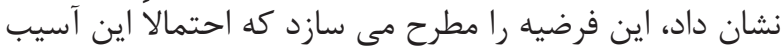

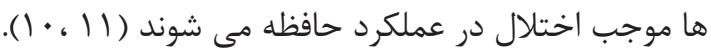
از سوى ديخر تجمع كلسيم در درون نورون ها كه به دنبال ايجاد

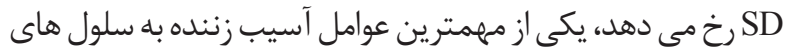

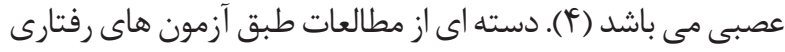

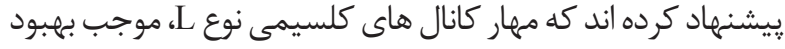

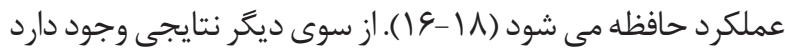

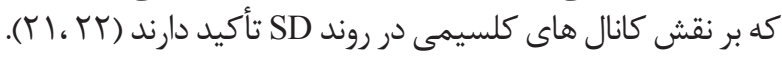

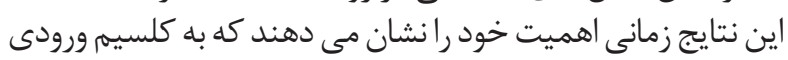

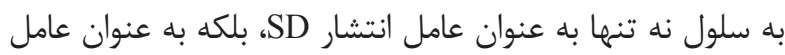

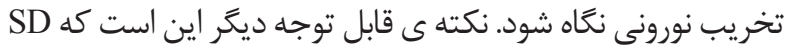

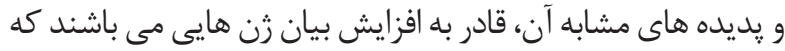

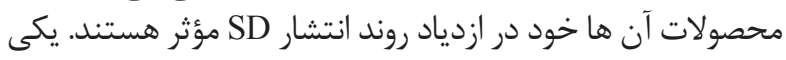

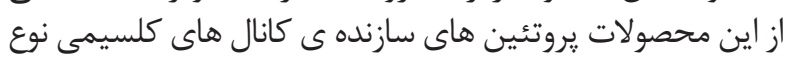

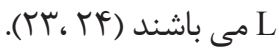

Yagami

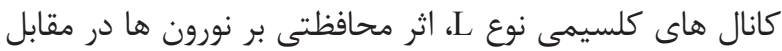

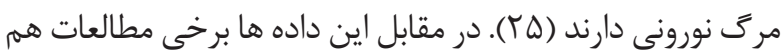

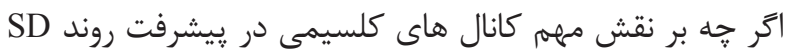


1. Leão AAP. Spreading depression of activity in the cerebral cortex. J Neurophysiol. 1944; 7: 359-90.

2. Lauritzen M, Dreier JP, Fabricius M, Hartings JA, Graf $\mathrm{R}$, Strong AJ. Clinical relevance of cortical spreading depression in neurological disorders: migraine, malignant stroke, subarachnoid and intracranial hemorrhage, and traumatic brain injury. J Cereb Blood Flow Metab. 2011; 31(1):17-35.

3. Bures J, Buresova O, Krivanek J. The mechanism and applications of Leao's spreading depression of EEG activity. New York, Academic, 1974.

4. Somjen GG. Mechanisms of spreading depression and hypoxic spreading depression-like depolarization. Physiol Rev. 2001; 81(3): 1065-96.

5. Gorji A .Spreading depression: a review of the clinical relevance. Brain Res Brain Res Rev. 2001; 38(1-2): 33-60.

6. Martins-Ferreira H, Nedergaard M, Nicholson C. Perspectives on spreading depression. Brain Res Brain Res Rev. 2000; 32: 215-34.

7. Canals S, Makarova I, López-Aguado L, Largo C, Ibarz JM, Herreras O. Longitudinal depolarization gradients along the somatodendritic axis of CA1 pyramidal cells: a novel feature of spreading depression. J Neurophysiol. 2005; 94: 943-51.

8. Dreier JP. The role of spreading depression, spreading depolarization and spreading ischemia in neurological disease. Nat Med. 2011; 17: 439-47.

9. Nedergaard M. Direct signaling from astrocytes to neurons in cultures of mammalian brain cells. Science. 1994; 263: 1768-71

10. Jafarian M, Rahimi S, Behnam F, Hosseini M, Haghir H, Sadeghzadeh B, et al. The effect of repetitive spreading depression on neuronal damage in juvenile rat brain. Neuroscience. 2010; 169(1): 388-94.

11. Sadeghian H, Jafarian M, Karimzadeh F, Kafami L, Kazemi H, Coulon P, et al. Neuronal death by repetitive cortical spreading depression in juvenile rat brain. Exp Neurol. 2012; 233(1): 438-46.

12. Egan MF, Kojima M, Callicott JH, Goldberg TE, Kolachana BS, Bertolino A, et al. The BDNF val66met polymorphism affects activity-dependent secretion of BDNF and human memory and hippocampal function. Cell. 2003; 112(2): 257-69.
13. Eichenbaum H. Hippocampus: Mapping or memory? Curr Biol. 2000; 10: 785-7.

14. Burešová $\mathrm{O}$, Bureš J. The effect of prolonged cortical spreading depression on learning and memory in rats. J Neurobiol. 1969; 1: 135-46.

15. Winn Jr, Kent MA, Libkuman TM. Learned taste aversion induced by cortical spreading depression. Physiol Behav. 1975; 15: 21-4.

16. Quartermain D, Garcia deSoria V. The effects of calcium channel antagonists on short-and long-term retention in mice using spontaneous alternation behavior. Neurobiol Learn Mem. 2001; 76: 117-24.

17. Quartermain D, desoria VG, Kwan A. Calcium channel antagonists enhance retention of passive avoidance and maze learning in mice. Neurobiol Learn Mem. 2001; 75: 77-90.

18. Quevedo J, Vianna M, Daroit D, Born AG, Kuyven CR, Roesler R, et al. L-type voltage-dependent calcium channel blocker nifedipine enhances memory retention when infused into the hippocampus. Neurobiol Learn Mem. 1998; 69(3): 320-5.

19. Maurice T, Bayle J, Privat A. Learning impairment following acute administration of the calcium channel antagonist nimodipine in mice. Behav Pharmacol. 1995; 6(2): 167-75.

20. Costa-Cruz RRG, Amâncio-dos-Santos Â, Guedes RCA. Characterization of cortical spreading depression in adult well-nourished and malnourished rats submitted to the association of pilocarpine-induced epilepsy plus streptozotocin-induced hyperglycemia. Neurosci Lett. 2006; 401: 271-5.

21. Jing J, Aitken PG, Somjen GG. Role of calcium channels i n spreading depression in rat hippocampal slices. Brain Res. 1993; 604: 251-9.

22. Richter F, Ebersberger A, Schaible H-G. Blockade of voltage-gated calcium channels in rat inhibits repetitive cortical spreading depression. Neurosci Lett. 2002; 334: 123-6.

23. Choudhuri R, Cui L, Yong C, Bowyer S, Klein RM, Welch KM, et al. Cortical spreading depression and gene regulation: relevance to migraine. Ann Neurol. 2002; 51: 499-506.

24. Westenbroek RE, Bausch SB, Lin RC, Franck JE, 
Noebels JL, Catterall WA. Upregulation of L-type $\mathrm{Ca} 2+$ channels in reactive astrocytes after brain injury, hypomyelination, and ischemia. J Neurosci. 1998; 18: 2321-34.

25. Yagami T, Ueda K, Sakaeda T, Itoh N, Sakaguchi G, Okamura N, et al. Protective effects of a selective L-type voltage-sensitive calcium channel blocker, S-312-d, on neuronal cell death. Biochem Pharmacol. 2004; 67(6): 1153-65.

26. Tottene A, Urbani A, Pietrobon D. Role of different voltage-gated $\mathrm{Ca}^{2+}$ channels in cortical spreading depression. Channels. 2011; 5: 110-4. 